\title{
Cold Stress Response: An Overview in Chlamydomonas
}

\author{
Elena Ermilova* \\ Biological Faculty, Saint-Petersburg State University, Saint-Petersburg, Russia
}

OPEN ACCESS

Edited by:

Eric Ruelland,

UMR7618 Institut d'écologie et des sciences de l'environnement de Paris

(IEES), France

Reviewed by:

Claire Remacle,

University of Liège, Belgium Rafael Catalá Rodríguez,

Spanish National Research

Council, Spain

Jose Ignacio Garcia-Plazaola, University of the Basque Country,

Spain

*Correspondence:

Elena Ermilova

e.ermilova@spbu.ru

Specialty section:

This article was submitted to

Plant Abiotic Stress,

a section of the journal

Frontiers in Plant Science

Received: 04 June 2020

Accepted: 18 August 2020

Published: 03 September 2020

Citation:

Ermilova E (2020) Cold Stress

Response: An Overview in

Chlamydomonas.

Front. Plant Sci. 11:569437.

doi: 10.3389/fpls.2020.569437
Low temperature (or cold) is one of the major environmental factors that limit the growth and development of many plants. Various plant species have evolved complex mechanisms to adjust to decreased temperature. Mesophilic chlorophytes are a widely distributed group of eukaryotic photosynthetic organisms, but there is insufficient information about the key molecular processes of their cold acclimation. The best available model for studying how chlorophytes respond to and cope with variations in temperature is the unicellular green alga Chlamydomonas reinhardtii. Chlamydomonas has been widely used for decades as a model system for studying the fundamental mechanisms of the plant heat stress response. At present, unraveling novel coldregulated events in Chlamydomonas has attracted increasing research attention. This mini-review summarizes recent progress on low-temperature-dependent processes in the model alga, while information on other photosynthetic organisms (cyanobacteria and land plants) was used to strengthen generalizations or specializations of cold-induced mechanisms in plant evolution. Here, we describe recent advances in our understanding of cold stress response in Chlamydomonas, discuss areas of controversy, and highlight potential future directions in cold acclimation research.

Keywords: Chlamydomonas reinhardtii, low temperature, cold stress, acclimation, chlorophyta

\section{INTRODUCTION}

Plants encounter various abiotic stresses, and fluctuations in temperature are among the most common. The capacity to integrate temperature cues and respond appropriately is shared among all plant organisms, ranging from single-celled algae to land plants. Low temperature (or cold) is one of the major environmental factors that limit the growth and development of many plant species (Sanghera et al., 2011; da Cruz et al., 2013; Jeon and Kim, 2013; Chen et al., 2014). Higher plants respond to cold treatment through complex processes at different levels, including physiological modifications and changes in cell membrane lipid composition and concentrations of proteins and metabolites (Kazemi-Shahandashti and Maali-Amiri, 2018). Cold-impacted plants induce the 
expression of a variety of genes that are controlled by abscisic acid-dependent and -independent pathways (Shi and Yang, 2014). Mesophilic green algae are a widely distributed group of eukaryotic photosynthetic organisms, but there is insufficient information about key physiological and molecular processes of their cold acclimation. In fact, the global cellular responses used by chlorophytes and other microorganisms to survive at low temperatures have been extensively investigated in the past few years with a focus on psychrophilic species (Margesin and Miteva, 2011; Cvetkovska et al., 2017; Collins and Margesin, 2019). The psychrophiles are characterized by the combination of several traits and the distantly related representatives often employ universal adaptations to permanently low temperatures, such as cold-adapted proteins, increased membrane fluidity, antifreeze proteins, and cryoprotectants (Siddiqui et al., 2013). At present, novel omics approaches allow the acquisition of information on the important features of mesophilic green algae to thrive in cold environment (Barati et al., 2019).

The best available model for studying how mesophilic green algae respond to and cope with variations in temperature is Chlamydomonas reinhardtii. The sequenced genomes, genetic maps, many molecular tools, and a large indexed mutant library (Merchant et al., 2007; Li et al., 2016; Blaby and Blaby-Haas, 2017) have facilitated the collection of information about the molecular mechanisms underlying the responses of this alga to temperature stresses. Chlamydomonas has been studied for decades with regard to heat stress response (Schroda et al., 2015; Rütgers et al., 2017); the process of its acclimation to cold is less understood. The ability of Chlamydomonas to adapt to low temperature (i.e., cold acclimation) can be induced at or below $7^{\circ} \mathrm{C}$ (Maikova et al., 2016). Since temperatures below $3^{\circ} \mathrm{C}$ lead to vegetative cell death after a short period (Valledor et al., 2013), freezing stress responses have not been analyzed in this alga. Physiologically, the cold-induced response of Chlamydomonas manifests as an acclimation phase during which cell division completely stops immediately after the temperature downshift (Lapina et al., 2013). During the cold acclimation phase of many bacteria, archaea, and plants, several cold shock proteins are synthesized, which help the cells to adapt to low temperatures (Phadtare, 2004; Barria et al., 2013; KetoTimonen et al., 2016). In contrast to higher plants, Chlamydomonas contains a single cold shock domain protein (CSP), termed NAB1 (Phytozome 13, C. reinhardtii v.5.6), that is not essential for low temperature acclimation (Mussgnug et al., 2005; Sawyer et al., 2015). This implies that cold-specific responses in the alga may be mediated by different, as yet uncharacterized, regulatory mechanisms. At present, unraveling novel cold-regulated events and the underlying transcriptional regulation in Chlamydomonas has attracted increasing research attention (Zalutskaya et al., 2019; Li et al., 2020).

This mini-review summarizes recent progress on lowtemperature-dependent processes in the model mesophilic alga, while information on other photosynthetic organisms (cyanobacteria and land plants) has been used to strengthen generalizations and specializations in plant evolution. Finally, we conclude that exploring plants with different lifestyles could provide new insights into the evolutionary plasticity of coldinduced adaptation mechanisms.

\section{EFFECTS OF LOW TEMPERATURE ON DIFFERENT CELL TYPES IN CHLAMYDOMONAS LIFE CYCLE}

Within the Chlorophyta, certain genera predominantly form a lichen symbiosis where mycobiont provides suitable conditions for physiological activity and tolerance to stresses of the photosynthetic partner (Karsten and Holzinger, 2012; Míguez et al., 2017). Chlamydomonas reinhardtii is a free-living singlecelled, biflagellate green alga found in fresh water and soil (Harris, 2009). Vegetative cells are haploid (n) and occur as two mating types, mating type plus $\left(\mathrm{mt}^{+}\right)$and mating type minus $\left(\mathrm{mt}^{-}\right)$(Figure 1). These cells divide by mitosis when there are adequate environmental conditions (Harris, 2001). The minimal temperature for continued growth of Chlamydomonas averages $15-13^{\circ} \mathrm{C}$, depending on the media conditions (Maikova et al., 2016). Although cell division completely stops immediately after a temperature downshift (Lapina et al., 2013; Valledor et al., 2013), the cell size continues to increase slowly (Maikova et al., 2016), and the morphology of these cells changes in such a way as a decrease in the density of nucleolus, a change in the chloroplast shape, an increase in the thickness of the starch shell around the pyrenoid, and an increase in the size of vacuoles (Valledor et al., 2013). The expression of a set of genes associated with the cell cycle is downregulated ( $\mathrm{Li}$ et al., 2020). Notably, three YAK1coding transcripts, the homolog of which in yeast negatively controls cell proliferation, accumulate steadily during lowtemperature treatment ( $\mathrm{Li}$ et al., 2020). Therefore, to some extent, Chlamydomonas uses molecular mechanisms to cope with temperature from 15 to $0^{\circ} \mathrm{C}$, resulting in viable but not dividing cells. Now, the question is whether Chlamydomonas uses cold-adapted cells like cysts as an overwintering form in the natural environment, and if so, whether it can form palmelloids or aggregates (de Carpentier et al., 2019) as an additional mechanism that helps vegetative cells adapt to cold conditions. Similarly, Chlamydomonas nivalis and Haematococcus pluvialis in response to cold stress become cysts (de Carpentier et al., 2019). Interestingly, in some filamentous green algae (Klebsormidium), the cold treatment induces the formation of spores, which show a significantly less susceptibility to photodamage compared to vegetative filaments (Míguez et al., 2020).

In addition to asexual reproduction, Chlamydomonas can undergo a sexual cycle (Figure 1). In laboratory conditions, gametogenesis is induced by nitrogen depletion in the presence of light, and then gametes of opposite mating types can fuse to form diploid (2n) zygotes that can develop into highly resistant dormant zygospores (Beck and Haring, 1996; Harris, 2001). Importantly, zygospores can survive low temperatures, including freezing (Suzuki and Johnson, 2002). It would be 


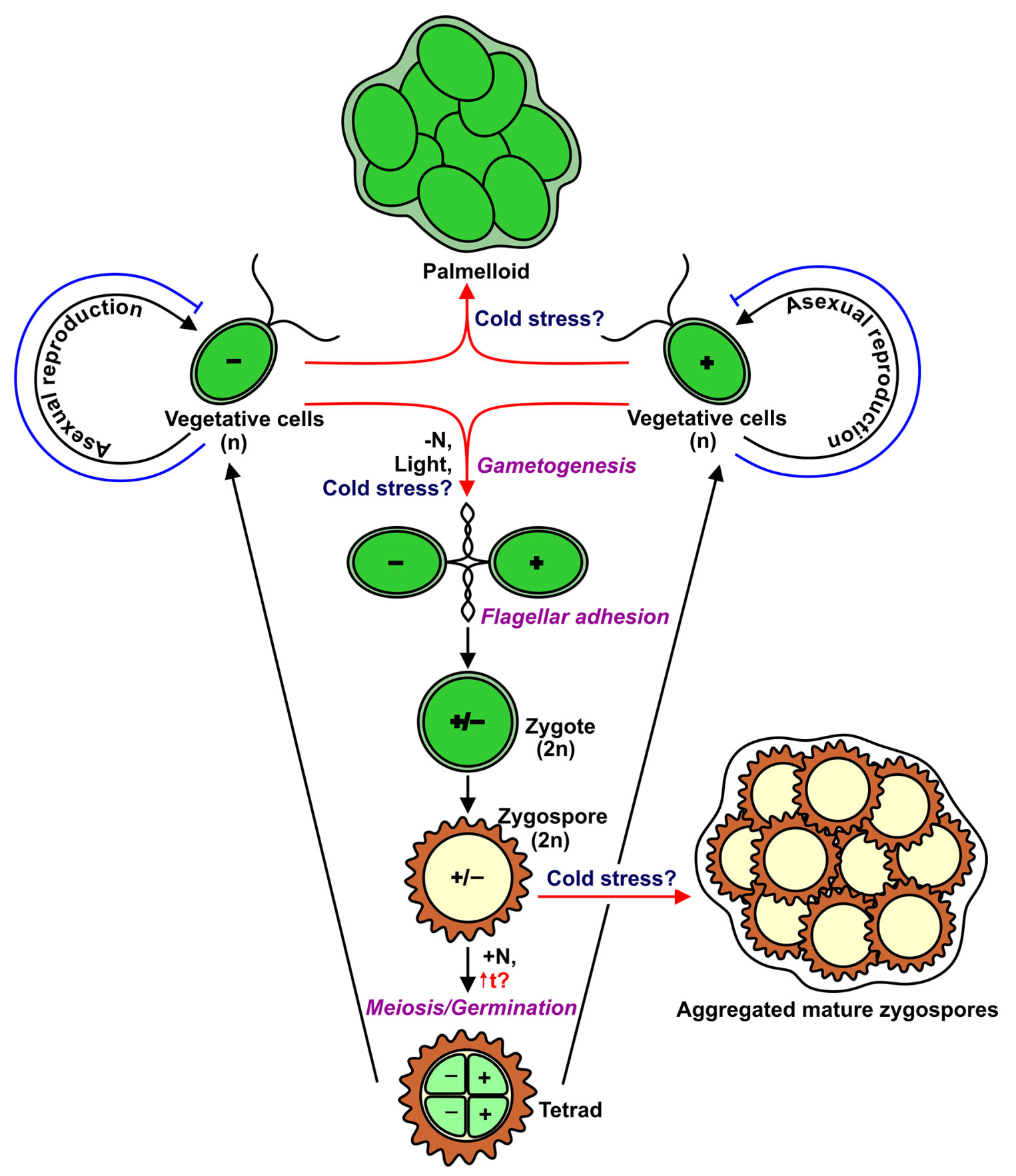

FIGURE 1 | Life cycle of Chlamydomonas with indication of multicellular structures such as palmelloids and aggregates. Red arrows show potential cold-induced pathways. Blue T-like lines indicate cold-induced block of cell division.

logical to hypothesize that the formation of Chlamydomonas zygospores is the most suitable strategy for cold adaptation. However, to form a diploid zygote, motile gametes of the opposite mating type should recognize each other through flagellar adhesion (Harris, 2001). Therefore, the flagella are essential players in the sexual cycle. After a temperature downshift, Chlamydomonas cells resorb their flagella for 4896 h (Valledor et al., 2013; Maikova et al., 2016). The most intriguing questions are which real signals trigger gametogenesis in the environment, and which cellular forms (vegetative cells or zygospores) are typical to survive low temperatures. 


\section{COLD-INDUCED SIGNALING MOLECULES AND REGULATORY PROTEINS}

The key to understanding plant cold response lies in identifying the possible molecular mechanisms of temperature sensing and signaling. Various photosynthetic organisms use different thermosensors to sense temperature changes, including membranes, proteins, nucleic acids, etc. (Sinetova and Los, 2016; Kazemi-Shahandashti and Maali-Amiri, 2018). One of the major direct consequences of temperature downshift is a decrease in membrane fluidity affecting membrane-associated cellular functions. In some bacteria, cold sensing occurs via changes in the membrane properties by cold sensor histidine kinases, such as DesK of Bacillus (Saita and de Mendoza, 2015) and Hik33 of Synechocystis (Murata and Los, 2006). In Chlamydomonas, to restore membrane flexibility, the amount of polyunsaturated fatty acids is increased (Valledor et al., 2013; Li et al., 2020). Although the exact sensor(s) for the perception of low temperature in this alga is still elusive, the membrane rigidification may be one of the potential mechanisms for activation of the downstream cold-induced signaling pathways (Figure 2). Interestingly, this process appears to be one of the first steps in cold stress signaling in Arabidopsis (Vaultier et al., 2006).
The next step is transduction of signals into biochemical processes via secondary messengers, such as $\mathrm{Ca}^{2+}$. In land plants, membrane rigidification can activate mechano-sensitive or ligand-activated $\mathrm{Ca}^{2+}$ channels, which leads to an increase in cytosolic $\mathrm{Ca}^{2+}$ and subsequent stimulation of the mitogenactivated protein kinase (MAPK) signaling pathway (Knight et al., 1996; Conde et al., 2011). Whether the cytosolic $\mathrm{Ca}^{2+}$ increases in response to cold stress in Clamydomonas remains to be determined. However, this concept is supported by the finding that transcripts encoding cyclic nucleotide-gated channel (CNGC)-like proteins and calcium-dependent protein kinases (CDPKs) accumulate in Chlamydomonas cells after cold treatment (Li et al., 2020). Other secondary messengers, cAMP and/or cGMP, can intertwine with calcium responses to mediate cold-induced signal transduction in the alga (Li et al., 2020).

Cold stress also induces $\mathrm{H}_{2} \mathrm{O}_{2}$ accumulation in plants, including Chlamydomonas (O'Kane et al., 1996; Zalutskaya et al., 2019). In various plants, $\mathrm{H}_{2} \mathrm{O}_{2}$ is a key signal molecule that mediates a series of responses (Desikan et al., 2004; Kazemi-Shahandashti and MaaliAmiri, 2018). In Chlamydomonas, expression of the cold-responsive gene GPX3 appears to be regulated by $\mathrm{H}_{2} \mathrm{O}_{2}$ (Zalutskaya et al., 2019). Interestingly, $\mathrm{H}_{2} \mathrm{O}_{2}$ can work in coordination with nitric oxide (NO) in plant cells (Neill et al., 2002; Thakur and Nayyar, 2013). Recent reports indicate that, in diverse plant species, NO

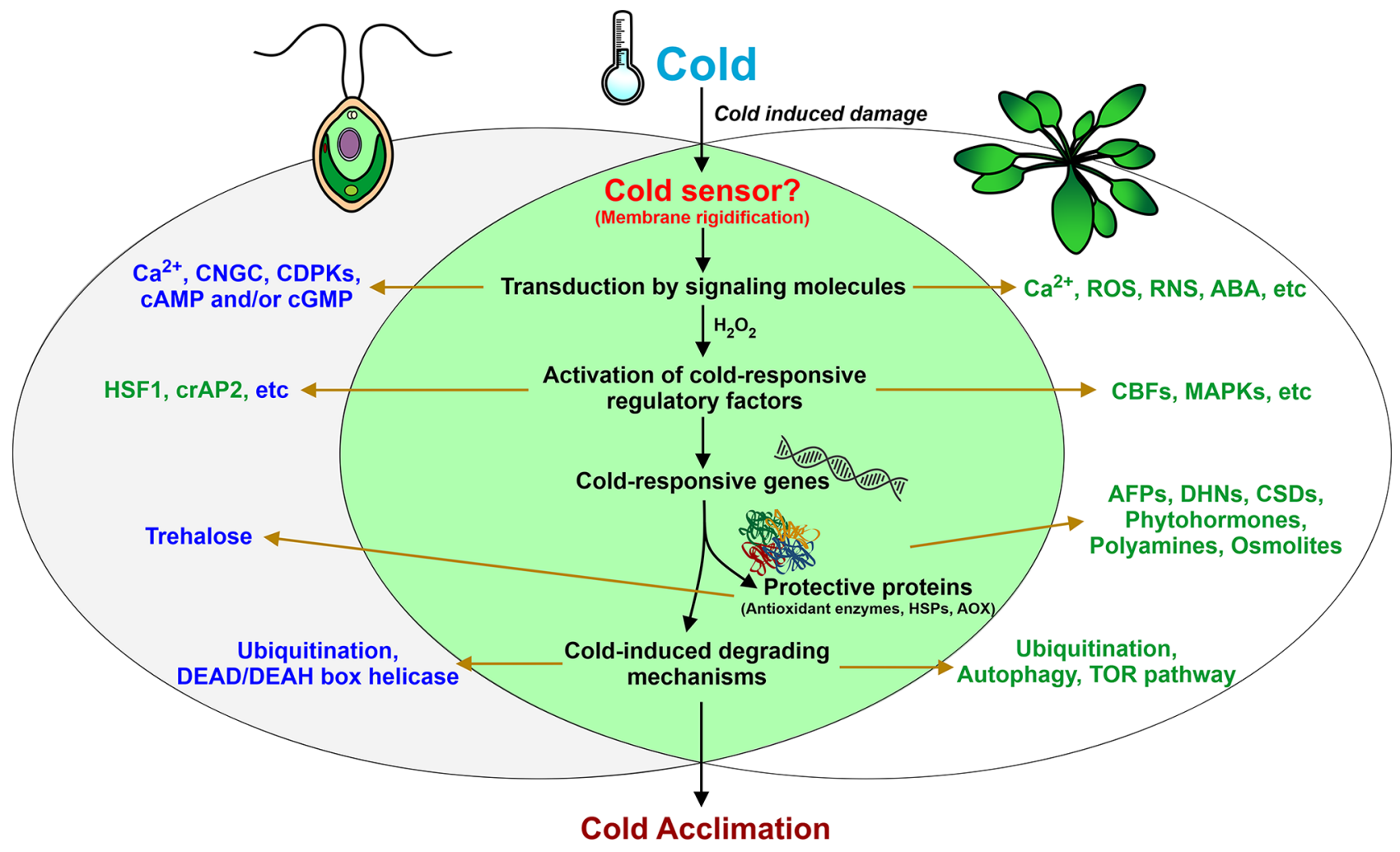

FIGURE 2 | Summary of fundamental responses of Chlamydomonas during low-temperature exposure. Left and right circles represent key cold-induced components of Chlamydomonas and land plants, respectively. Proven characterized components common to Chlamydomonas and land plants are shown in the intersection of two black circles. Components and processes that are potentially involved in Chlamydomonas cold acclimation but have been characterized only on the gene expression level are shown in blue. Other proven components are shown in blue. 
plays a crucial role in the control of cold-responsive gene transcription and in post-translational modifications of coldresponsive proteins, namely S-nitrosylation (Puyaubert and Baudouin, 2014). Additionally, in cold-exposed plants, the enhancement of antioxidant defense is achieved via different NOdependent regulatory mechanisms (Farnese et al., 2016). NO is associated with many signaling processes of Chlamydomonas (Hemschemeier et al., 2013; Wei et al., 2014; Filina et al., 2019). However, the role of NO in cold signaling in Chlamydomonas has not yet been explored.

In cold-stressed plants, there are two main groups of regulatory proteins; those that catalyze dephosphorylation and transcription factors (TFs) (Chinnusamy et al., 2006; Lissarre et al., 2010; Kazemi-Shahandashti and Maali-Amiri, 2018). As mentioned above, CNGC- and CDPK-coding genes are cold responsive, suggesting the potential involvement of these kinases in cold-induced signaling (Li et al., 2020). Moreover, the plant-specific serine/threonine kinase SnRK2.2, which has a key role in sulfur deprivation responses (Gonzalez-Ballester et al., 2008), is believed to also be involved in the cold acclimation process of Chlamydomonas (Unal and Cekic, 2019).

Among the important targets of kinase cascades triggered by cold shock is HSF1 (Maikova et al., 2016), which is a central regulator of the heat stress response in Chlamydomonas (SchulzRaffelt et al., 2007). Another potential TF of regulatory networks in Chlamydomonas under low temperature is cold-inducible crAP2 (Li et al., 2020), which belongs to the AP2/EREBP family (Xie et al., 2019). Many TFs from different superfamilies are potential candidates as cold response regulators ( $\mathrm{Li}$ et al., 2020). However, further studies are required to fully understand which of these represent components of the regulatory networks in cold stress.

\section{IMPACTS OF COLD ON FEATURES RELATED TO ENERGY GENERATION}

Low-temperature stress affects many aspects of photosynthesis in land plants (Huner et al., 1998; Ensminger et al., 2006; Hajihashemi et al., 2018; Zhu et al., 2018) and green algae (Míguez et al., 2017; Míguez et al., 2020; Zheng et al., 2020). In terrestrial green algae, down-regulation of photochemical processes is a controlled mechanism that favors the dissipation of excess energy to protect the photosynthetic apparatus under low temperature conditions (Míguez et al., 2017). In Chlamydomonas, after a temperature decrease, some of the light harvesting complex proteins and $b_{6} f$ cytochromes decline, leading to diminished photosynthetic capacity (Valledor et al., 2013). Additionally, although enzymes belonging to the $\mathrm{CO}_{2}$ fixation pathway remain at the same level during cold treatment, impaired expression and accumulation of Rubisco and subsequent decreased activity of the Calvin cycle may contribute to overreduction-associated damage to the photosystem and inhibition of the photosynthetic rate (Hashida and Kawai-Yamada, 2019).
In contrast, transcripts of light harvesting chlorophyll $\mathrm{a} / \mathrm{b}$ stressrelated binding protein LHCSR1, which is responsible for nonphotochemical quenching (NPQ) in cells and the PSI-dependent photoprotection mechanism (Kosuge et al., 2018), accumulate during cold exposure (Li et al., 2020). Since NPQ is not decreased in coldtreated C. reinhardtii (Zheng et al., 2020), LHCSR1 can also protect the photosynthetic apparatus under low temperatures. Additionally, in cold-treated Chlamydomonas, VIPP1, thylakoid formation protein (THF), and monogalactosyldiacylglycerol-specific lipase PGD1 (PLASTID GALACTOGLYCEROLIPID DEGRADATION1) are accumulated (Valledor et al., 2013; Du et al., 2018). VIPP1 and PGD1 appeared to be involved in the organization of thylakoid membranes, where the biogenesis/repair at least of PSII might occur (Rütgers and Schroda, 2013; Du et al., 2018). Interestingly, the enhanced abundance of several photosynthesis-related proteins has been reported under cold stress in Arabidopsis, winter wheat, and rice (Janmohammadi et al., 2015). The accumulation of such proteins with the partial recovery of adenosine triphosphate (ATP) synthesis proteins in Chlamydomonas suggests the presence of a potential mechanism to reestablish the features of the photosynthetic machinery present in the alga before the onset of cold stress. Further research is needed to validate this hypothesis.

Given that $\mathrm{O}_{2}$ solubility increases at low temperatures, increased levels of reactive oxygen species (ROS) are generated, which leads to oxidative stress. Like land plants, Chlamydomonas also generates $\mathrm{H}_{2} \mathrm{O}_{2}$ under hypothermia (Zalutskaya et al., 2019). For this reason, as in other unicellular organisms at low temperatures (Tribelli and López, 2018), in this alga, proteins involved in oxidative metabolism such as the tricarboxylic acid cycle (TCA) and the electron transport chain are repressed (Valledor et al., 2013). Notably, in land plants, the TCA is an intermediary and its related enzymes are controlled in an opposite way (Kaplan et al., 2007). On the other hand, glycolysis is maintained at the same level and two key enzymes involved in the pentose-phosphate pathway, 6-phosphogluconolactonase and transaldolase 2, demonstrate increased levels, indicating important features for energy generation in Chlamydomonas under cold temperatures (Valledor et al., 2013). No information is available regarding cold-mediated modification in enzymes of glycolysis and the TCA cycle. In spite of this information, additional studies on the consequences of low-temperature stress on glycolytic and respiratory metabolism in Chloroplastida are required.

Many plants degrade their starch reserves to provide energy, sugars, and derived metabolites to mitigate the negative effects of stress (Thalmann and Santelia, 2017). In contrast, in cold-treated Chlamydomonas cells, starch is constantly accumulated (Valledor et al., 2013). Furthermore, an increase in starch abundance under stress also occurs in Arabidopsis (Kaplan and Guy, 2004; Skirycz et al., 2010) and in its close relative Thellungiella halophila (Wang et al., 2013). Although the reason for this difference is unclear, increased starch content is mainly seen in response to high salinity or cold stress (Thalmann and Santelia, 2017). Future research should be directed toward understanding whether it is a species-specific or stressspecific feature. 


\section{DEFENSE MECHANISMS AND PROTECTIVE PROTEINS}

Due the imbalanced absorption and utilization of light induced by cold treatment, over-accumulation of ROS occurs in plants (O'Kane et al., 1996; Ensminger et al., 2006; Barati et al., 2019). Some cold-exposed green algae significantly increase the content of photoprotective pigments such as antheraxanthin, zeaxanthin, and total carotenes (Míguez et al., 2020). During cold adaptation, ROS-scavenging enzymes are essential for ROS detoxification (Suzuki and Mittler, 2006; Distelbarth et al., 2013). As in land plants, low-temperature stress induces $\mathrm{H}_{2} \mathrm{O}_{2}$ accumulation in Chlamydomonas (Zalutskaya et al., 2019), which may be a result of Rubisco side reactions and Mehler reactions, when sinks for electrons from photosynthetic light reactions become limited (Mehler, 1951).

Notably, among three classes of antioxidant enzymes abolishing the action of $\mathrm{H}_{2} \mathrm{O}_{2}$ [ascorbate peroxidases (APX), catalases (CAT), and glutathione peroxidases (GPX)], Chlamydomonas cells under hypothermia induce only GPX genes and likely use only one class of antioxidant enzymes for defense of different cell compartments during cold action (Zalutskaya et al., 2019).

Overreduction of the electron transport chain in mitochondria is also a major mechanism of ROS production during temperature stresses (Suzuki and Mittler, 2006). In contrast to animal mitochondria, those of plants possess a branched electron-transport chain with two terminal oxidases, the energy-conserving cytochrome oxidase and the differently termed alternative oxidase (AOX), which is not a proton pump (Affourtit et al., 2002; Finnegan et al., 2004; Millar et al., 2011). Plant AOX prevents overproduction of reactive oxygen species (Vanlerberghe, 2013). In Chlamydomonas, AOX1 is a coldinducible (Molen et al., 2006), suggesting a potential role of this accessory pathway in the hypothermia tolerance of the alga.

In land plants, cold-induced defense mechanisms also include activation of the synthesis of some cryoprotectants such as sugars, proline, glycine betaine, trehalose, and polyamines (Longo et al., 2018). Even though the coordination of biosynthetic gene expression and metabolite accumulation of sucrose and trehalose has been found (Valledor et al., 2013), their protective role in Chlamydomonas is questionable, since the increased abundance is small. Additionally, antioxidants such as ascorbic acid and glutathione are not involved in Chlamydomonas acclimation to cold stress (Valledor et al., 2013). Moreover, in contrast to other stresses, a shift to low temperature does not induce glycerin, proline, and putrescine synthesis in this alga (Lapina et al., 2013; Valledor et al., 2013). Therefore, whether organic protective molecules are crucial parts of cold adaptation mechanisms in Chlamydomonas needs to be investigated.

Another interesting feature of Chlamydomonas's response to temperature downshift is that cold-shock domain nucleic acid binding proteins are not associated with the development of cold tolerance in cells (Mussgnug et al., 2005; Sawyer et al., 2015). These cold-shock proteins function as RNA chaperones (Graumann and
Marahiel, 1998; Phadtare, 2004). Whether other proteins exist in algal cells to maintain mRNA in a linear single-stranded form prior to translation remains an open question.

Low temperatures also cause DNA damage in plants (Thakur and Nayyar, 2013). When exposed to cold stress, Chlamydomonas may promote cell cycle arrest and DNA repair in order to avoid DNA damage (Li et al., 2020).

In all organisms, the molecular basis of the responses to many different stresses includes rapid accumulation of heat shock proteins (HSPs). Also, in land plants and Chlamydomonas, HSPs accumulate in response to low temperatures (Renaut et al., 2006; Timperio et al., 2008; Maikova et al., 2016). Whether other protein families (for example, antifreeze or dehydrin proteins) play a role in the defense mechanisms of Chlamydomonas at low temperatures is still unknown (Figure 2). Interestingly, Chlamydomonas was used to express and secrete a functional antifreeze protein from Lolium perenne (Gramineae) (Lauersen et al., 2013).

\section{COLD-INDUCED DEGRADING MECHANISMS}

One of the important consequences of temperature downshift on plants is protein degradation (Kazemi-Shahandashti and MaaliAmiri, 2018). This degradation is essential for the breakdown of misfolded proteins and, as a result, for the production of amino acids and peptides, which can be used to synthesize new proteins that are necessary for adaptation to cold.

Ubiquitination is an important step in nonlysosomal protein degradation in all eukaryotes. Misfolded proteins that are labeled by ubiquitins can then be acted on by proteolytic enzymes called proteasomes. In Chlamydomonas, the cold-induced accumulation of RING/U-box superfamily protein (Li et al., 2020) and other coldspecific changes in the ubiquitination system (Valledor et al., 2013) suggest that ubiquitination is involved in cold adaptation. The degradation of abnormal proteins through ubiquitination has also been shown in cold-stressed land plants (Heidarvand and MaaliAmiri, 2013).

Another highly conservative protein degradation system is autophagy (Liu and Bassham, 2012; Michaeli et al., 2016). This catabolic process is mediated by autophagy-related proteins, which have been identified in various organisms, including Chlamydomonas (Pérez-Pérez et al., 2010; Pérez-Pérez and Crespo, 2010). In plants, autophagy participates in the responses to many environmental stresses (Wang et al., 2017). Although in Chlamydomonas, autophagy is also induced under different stresses (Pérez-Pérez et al., 2017), the potential mechanisms underlying cold-stress-dependent activation of autophagy are still poorly understood (Valledor et al., 2013).

Temperature downshift also leads to changes in secondary structures of RNA and the formation of defective RNA. In Chlamydomonas, in the absence of cold-induced CSPs (Mussgnug et al., 2005), RNA helicases and exoribonucleases might stimulate RNA degradation at low temperatures. Indeed, accumulation of the DEAD/DEAH box helicase occurs in algal cells upon cold stress 
(Valledor et al., 2013). Interestingly, in land plants, members of the $\mathrm{DEAD} / \mathrm{DEAH}$ box helicase family enhance tolerance to stresses, including cold treatment (Gong et al., 2002; Gong et al., 2005; Vashisht and Tuteja, 2006). Additionally, RNA degradation mediated by exoribonucleases affects plant responses to stresses (Merret et al., 2013). However, unlike bacteria (Phadtare and Severinov, 2010), the role of exoribonucleases in the cold acclimation of plants is still unknown.

\section{CONCLUSION AND FUTURE PERSPECTIVES}

Chlamydomonas serves as a model plant system to examine many aspects of heat stress response and recently has been used in studies of cold-induced responses; the latter studies have shown that Chlamydomonas contains both mechanisms common to other plants and species-specific mechanisms of cold tolerance. Though much work has been done, a lot remains to be done in order to unravel the cold sensor(s) and whole mechanism of signaling and its control. Most of the transcription factors and regulatory proteins for cold-induced signaling network in this alga, often inferred from transcriptomic and metabolomics studies, have not been characterized biochemically. Furthermore, generating and analyzing the molecular genetics of cold-sensitive or cold-tolerant Chlamydomonas strains that underexpress or overexpress one or more genes will provide us with an understanding of the basic mechanism of the functioning of stress genes during lowtemperature exposure. An outstanding question is related to the

\section{REFERENCES}

Affourtit, C., Albury, M. S., Crichton, P. G., and Moore, A. L. (2002). Exploring the molecular nature of alternative oxidase regulation and catalysis. FEBS Lett. 510, 121-126. doi: 10.1016/S0014-5793(01)03261-6

Barati, B., Gan, S.-Y., Lim, P.-E., Beardall, J., and Phang, S.-M. (2019). Green algal molecular responses to temperature stress. Acta Physiol. Plant 41, 26. doi: 10.1007/s11738-019-2813-1

Barria, C., Malecki, M., and Arraiano, C. M. (2013). Bacterial adaptation to cold. Microbiol 159, 2437-2443. doi: 10.1099/mic.0.052209-0

Beck, C. F., and Haring, M. A. (1996). Gametic differentiation of Chlamydomonas. Int. Rev. Cytol. 168, 259-302. doi: 10.1016/S0074-7696(08)60886-4

Blaby, I. K., and Blaby-Haas, C. E. (2017). "Genomics and Functional Genomics in Chlamydomonas reinhardtii," in Chlamydomonas: Molecular Genetics and Physiology. Ed. M. Hippler (Cham: Springer), 30, 1-26. Microbiology Monographs. doi: 10.1007/978-3-319-66365-4_1

Chen, L.-J., Xiang, H.-Z., Miao, Y., Zhang, L., Guo, Z.-F., Zhao, X.-H., et al. (2014). An overview of cold resistance in plants. J. Agron. Crop Sci. 200, 237-245. doi: 10.1111/j.1399-3054.2006.00596.x

Chinnusamy, V., Zhu, J., and Zhu, J. K. (2006). Gene regulation during cold acclimation in plants. Physiol. Plant 126, 52-61. doi: 10.1111/j.13993054.2006.00596.x

Collins, T., and Margesin, R. (2019). Psychrophilic lifestyles: mechanisms of adaptation and biotechnological tools. Appl. Microbiol. Biotechnol. 103 (7), 2857-2871. doi: 10.1007/s00253-019-09659-5

Conde, A., Chaves, M. M., and Gerós, H. (2011). Membrane transport, sensing and signaling in plant adaptation to environmental stress. Plant Cell Physiol. 52, 1583-1602. doi: 10.1093/pcp/pcr107

Cvetkovska, M., Hüner, N. P. A., and Smith, D. R. (2017). Chilling out: the evolution and diversification of psychrophilic algae with a focus on relationship between the intensity of the cold (chilling or freezing) and the potential strategies used by this alga in nature (cold-adapted vegetative cells, dormant zygospores, palmelloids, or aggregates). Another interesting avenue will be comparative genomics of psychrophilic Chlamydomonas (such as Chlamydomonas sp. UWO241, Chlamydomonas sp. ICE-L, Chlamydomonas nivalis) with that of a representative mesophilic to reveal the unique components or mechanisms present or absent from psychrophiles. We are only starting to have a sense of this, and clearly there is a lot more to learn.

\section{AUTHOR CONTRIBUTIONS}

The author confirms being the sole contributor of this work and has approved it for publication.

\section{FUNDING}

This work was supported by a grant from the Russian Science Foundation (grant no.16-14-10004) to EE.

\section{ACKNOWLEDGMENTS}

The author is grateful to Vitalina Derkach and Alexandra Grinko (Saint-Petersburg State University) for technical assistance.

Chlamydomonadales. Polar. Biol. 40, 1169-1184. doi: 10.1007/s00300-0162045-4

da Cruz, R. P., Sperotto, R. A., Cargnelutti, D., Adamski, J. M., de FreitasTerra, T., and Fett, J. P. (2013). Avoiding damage and achieving cold tolerance in rice plants. Food Energy Secur. 2, 96-119. doi: 10.1002/fes3.25

de Carpentier, F., Lemaire, S. D., and Danon, A. (2019). When unity is strength: the strategies used by Chlamydomonas to survive environmental stresses. Cells 8, 1307. doi: 10.3390/cells8111307

Desikan, R., Hancock, J. T., and Neill, S. J. (2004). "Oxidative stress signalling," in Plant Responses to Abiotic Stress. Topics in Current Genetics. Eds. H. Hirt and K. Shinozaki (Berlin, Heidelberg: Springer), 121-149. doi: 10.1007/978-3-54039402-0_6

Distelbarth, H., Nägele, T., and Heyer, A. G. (2013). Responses of antioxidant enzymes to cold and high light are not correlated to freezing tolerance in natural accessions of Arabidopsis thaliana. Plant Biol. 15, 982-990. doi: 10.1111/j.1438-8677.2012.00718.x

Du, Z. Y., Lucker, B. F., Zienkiewicz, K., Miller, T. E., Zienkiewicz, A., Sears, B. B., et al. (2018). Galactoglycerolipid lipase PGD1 is involved in thylakoid membrane remodeling in response to adverse environmental conditions in. Chlamydomonas Plant Cell 30, 447-465. doi: 10.1105/tpc.17.00446

Ensminger, I., Busch, F., and Huner, N. P. A. (2006). Photostasis and cold acclimation: sensing low temperature through photosynthesis. Physiol. Plant 126, 28-44. doi: 10.1111/j.1399-3054.2006.00627.x

Farnese, F. S., Menezes-Silva, P. E., Gusman, G. S., and Oliveira, J. A. (2016). When Bad Guys Become Good Ones: The Key Role of Reactive Oxygen Species and Nitric Oxide in the Plant Responses to Abiotic Stress. Front. Plant Sci. 7, 471. doi: 10.3389/fpls.2016.00471

Filina, V., Grinko, A., and Ermilova, E. (2019). Truncated hemoglobins 1 and 2 are implicated in the modulation of phosphorus deficiency-induced nitric oxide levels in Chlamydomonas. Cells 8, 947. doi: 10.3390/cells8090947 
Finnegan, P. M., Soole, K. L., and Umbach, A. L. (2004). "Alternative mitochondrial electron transport proteins in higher plants," in Plant Mitochondria: From Genome to Function. Advances in Photosynthesis and Respiration. Eds. D. A. Day, A. H. Millar and J. Whelan (Dordrecht: Springer Netherlands), 163-230. doi: 10.1007/978-1-4020-2400-9_9

Gong, Z., Lee, H., Xiong, L., Jagendorf, A., Stevenson, B., and Zhu, J.-K. (2002). RNA helicase-like protein as an early regulator of transcription factors for plant chilling and freezing tolerance. PNAS 99, 11507-11512. doi: 10.1073/pnas.172399299

Gong, Z., Dong, C.-H., Lee, H., Zhu, J., Xiong, L., Gong, D., et al. (2005). A DEAD box RNA helicase is essential for mRNA export and important for development and stress responses in Arabidopsis. Plant Cell. 17, 256-267. doi: $10.1105 /$ tpc.104.027557

Gonzalez-Ballester, D., Pollock, S. V., Pootakham, W., and Grossman, A. R. (2008). The central role of a SNRK2 kinase in sulfur deprivation responses. Plant Physiol. 147, 216-227. doi: 10.1104/pp.108.116137

Graumann, P. L., and Marahiel, M. A. (1998). A superfamily of proteins that contain the cold-shock domain. Trends Biochem. Sci. 23, 286-290. doi: 10.1016/S0968-0004(98)01255-9

Hajihashemi, S., Noedoost, F., Geuns, J. M. C., Djalovic, I., and Siddique, K. H. M. (2018). Effect of cold stress on photosynthetic traits, carbohydrates, morphology, and anatomy in nine cultivars of Stevia rebaudiana. Front. Plant Sci. 9, 1430. doi: 10.3389/fpls.2018.01430

Harris, E. H. (2001). Chlamydomonas as a model organism. Annu. Rev. Plant Physiol. Plant Mol. Biol. 52, 363-406. doi: 10.1146/annurev.arplant.52.1.363

Harris, E. H. (2009). The Chlamydomonas sourcebook: introduction to Chlamydomonas and its laboratory use (San Diego: Academic Press).

Hashida, S., and Kawai-Yamada, M. (2019). Inter-organelle NAD metabolism underpinning light responsive NADP dynamics in plants. Front. Plant Sci. 10, 960. doi: $10.3389 /$ fpls.2019.00960

Heidarvand, L., and Maali-Amiri, R. (2013). Physio-biochemical and proteome analysis of chickpea in early phases of cold stress. J. Plant Physiol. 170, 459469. doi: 10.1016/j.jplph.2012.11.021

Hemschemeier, A., Düner, M., Casero, D., Merchant, S. S., Winkler, M., and Happe, T. (2013). Hypoxic survival requires a 2-on-2 hemoglobin in a process involving nitric oxide. PNAS 110, 10854-10859. doi: 10.1073/pnas.1302592110

Huner, N. P. A., Öquist, G., and Sarhan, F. (1998). Energy balance and acclimation to light and cold. Trends Plant Sci. 3, 224-230. doi: 10.1016/S1360-1385(98)01248-5

Janmohammadi, M., Zolla, L., and Rinalducci, S. (2015). Low temperature tolerance in plants: Changes at the protein level. Phytochemistry 117, 76-89. doi: 10.1016/j.phytochem.2015.06.003

Jeon, J., and Kim, J. (2013). Cold stress signaling networks in Arabidopsis. J. Plant Biol. 56, 69-76. doi: 10.1007/s12374-013-0903-y

Kaplan, F., and Guy, C. L. (2004). $\beta$-amylase induction and the protective role of maltose during temperature shock. Plant Physiol. 135, 1674-1684. doi: 10.1104/pp.104.040808

Kaplan, F., Kopka, J., Sung, D. Y., Zhao, W., Popp, M., Porat, R., et al. (2007). Transcript and metabolite profiling during cold acclimation of Arabidopsis reveals an intricate relationship of cold-regulated gene expression with modifications in metabolite content. Plant J. 50, 967-981. doi: 10.1111/ j.1365-313X.2007.03100.x

Karsten, U., and Holzinger, A. (2012). Light, temperature and desiccation effects on photosynthetic activity, and drought-induced ultrastructural changes in thegreen alga Klebsormidium disectum (Streptophyta) from a high alpine soil crust. Microb. Ecol. 63, 51-63. doi: 10.1007/s00248-011-9924-6

Kazemi-Shahandashti, S.-S., and Maali-Amiri, R. (2018). Global insights of protein responses to cold stress in plants: Signaling, defence, and degradation. J. Plant Physiol. 226, 123-135. doi: 10.1016/j.jplph.2018.03.022

Keto-Timonen, R., Hietala, N., Palonen, E., Hakakorpi, A., Lindström, M., and Korkeala, H. (2016). Cold shock proteins: a minireview with special emphasis on Csp-family of enteropathogenic Yersinia. Front. Microbiol. 7, 1151. doi: $10.3389 /$ fmicb.2016.01151

Knight, H., Trewavas, A. J., and Knight, M. R. (1996). Cold calcium signaling in Arabidopsis involves two cellular pools and a change in calcium signature after acclimation. Plant Cell. 8, 489-503. doi: 10.1105/tpc.8.3.489

Kosuge, K., Tokutsu, R., Kim, E., Akimoto, S., Yokono, M., Ueno, Y., et al. (2018). LHCSR1-dependent fluorescence quenching is mediated by excitation energy transfer from LHCII to photosystem I in Chlamydomonas reinhardtii. PNAS 115, 3722-3727. doi: 10.1073/pnas.1720574115
Lapina, T., Zalutskaya, Z., Anikina, A., and Ermilova, E. (2013). Accumulation and export of glycerol in unicellular green alga Chlamydomonas reinhardtii. Biol. Commun. 2, 36-40.

Lauersen, K. J., Vanderveer, T. L., Berger, H., Kaluza, I., Mussgnug, J. H., Walker, V. K., et al. (2013). Ice recrystallization inhibition mediated by a nuclearexpressed and -secreted recombinant ice-binding protein in the microalga. Chlamydomonas Reinhardtii. Appl. Microbiol. Biotechnol. 97 (22), 9763-9772. doi: 10.1007/s00253-013-5226-x

Li, X., Zhang, R., Patena, W., Gang, S. S., Blum, S. R., Ivanova, N., et al. (2016). An indexed, mapped mutant library enables reverse genetics studies of biological processes in Chlamydomonas reinhardtii. Plant Cell 28, 367-387. doi: 10.1105/ tpc. 15.00465

Li, L., Peng, H., Tan, S., Zhou, J., Fang, Z., Hu, Z., et al. (2020). Effects of early cold stress on gene expression in Chlamydomonas reinhardtii. Genomics 112, 11281138. doi: 10.1016/j.ygeno.2019.06.027

Lissarre, M., Ohta, M., Sato, A., and Miura, K. (2010). Cold-responsive gene regulation during cold acclimation in plants. Plant Signal. Behav. 5, 948-952. doi: $10.4161 /$ psb.5.8.12135

Liu, Y., and Bassham, D. C. (2012). Autophagy: pathways for self-eating in plant cells. Annu. Rev. Plant Biol. 63, 215-237. doi: 10.1146/annurev-arplant042811-105441

Longo, V., Janmohammadi, M., Zolla, L., and Rinalducci, S. (2018). "What can small molecules tell us about cold stress tolerance in plants?," in Cold Tolerance in Plants: Physiological, Molecular and Genetic Perspectives. Eds. S. H. Wani and V. Herath (Cham: Springer), 127-157. doi: 10.1007/978-3-030-01415-5_8

Maikova, A., Zalutskaya, Z., Lapina, T., and Ermilova, E. (2016). The HSP70 chaperone machines of Chlamydomonas are induced by cold stress. J. Plant Physiol. 204, 85-91. doi: 10.1016/j.jplph.2016.07.012

Margesin, R., and Miteva, V. (2011). Diversity and ecology of psychrophilic microorganisms. Res. Microbiol. 162, 346e361. doi: 10.1016/j.resmic. 2010.12.004

Mehler, A. H. (1951). Studies on reactions of illuminated chloroplasts. II. Stimulation and inhibition of the reaction with molecular oxygen. Arch. Biochem. Biophys. 34, 339-351. doi: 10.1016/0003-9861(51)90082-3

Merchant, S. S., Prochnik, S. E., Vallon, O., Harris, E. H., Karpowicz, S. J., Witman, G. B., et al. (2007). The Chlamydomonas genome reveals the evolution of key animal and plant functions. Science 318, 245-250. doi: 10.1126/ science. 1143609

Merret, R., Descombin, J., Juan, Y., Favory, J.-J., Carpentier, M.-C., Chaparro, C., et al. (2013). XRN4 and LARP1 are required for a heat-triggered mRNA decay pathway involved in plant acclimation and survival during thermal stress. Cell Rep. 5, 1279-1293. doi: 10.1016/j.celrep.2013.11.019

Michaeli, S., Galili, G., Genschik, P., Fernie, A. R., and Avin-Wittenberg, T. (2016). Autophagy in plants - what's new on the menu? Trends Plant Sci. 21, 134-144. doi: 10.1016/j.tplants.2015.10.008

Míguez, F., Schiefelbein, U., Karsten, U., García-Plazaola, J. I., and Gustavs, L. (2017). Unraveling the photoprotectiv response of lichenized and free-living green algae (Trebouxiophyceae, Chlorophyta) to photochilling stress. Front. Plant Sci. 8, 1144. doi: 10.3389/fpls.2017.01144

Míguez, F., Holzinger, A., Fernandez-Marin, B., García-Plazaola, G. I., Karsten, U., and Gustavs, L. (2020). Ecophysiological changes and spore formation: two strategies in response to low-temperature and high-light stress in Klebsormidium cf. flaccidum (Klebsormidiophyceae, Streptophyta). J. Phycol. 15, 649-661. doi: 10.1111/jpy.12971-19-180

Millar, A. H., Whelan, J., Soole, K. L., and Day, D. A. (2011). Organization and regulation of mitochondrial respiration in plants. Annu. Rev. Plant Biol. 62, 79-104. doi: 10.1146/annurev-arplant-042110-103857

Molen, T. A., Rosso, D., Piercy, S., and Maxwell, D. P. (2006). Characterization of the alternative oxidase of Chlamydomonas reinhardtii in response to oxidative stress and a shift in nitrogen source. Physiol. Plant 127, 74-86. doi: 10.1111/ j.1399-3054.2006.00643.x

Murata, N., and Los, D. A. (2006). Histidine kinase Hik33 is an important participant in cold-signal transduction in cyanobacteria. Physiol. Plant 126, 17-27. doi: 10.1111/j.1399-3054.2006.00608.x

Mussgnug, J. H., Wobbe, L., Elles, I., Claus, C., Hamilton, M., Fink, A., et al. (2005). NAB1 is an RNA binding protein involved in the light-regulated differential expression of the light-harvesting antenna of Chlamydomonas reinhardtii. Plant Cell. 17, 3409-3421. doi: 10.1105/tpc.105.035774 
Neill, S. J., Desikan, R., Clark, A., Hurst, R. D., and Hancock, J. T. (2002). Hydrogen peroxide and nitric oxide as signaling molecules in plants. J. Exp. Bot. 53, 1237-1247. doi: 10.1093/jexbot/53.372.1237

O’Kane, D., Gill, V., Boyd, P., and Burdon, R. (1996). Chilling, oxidative stress and antioxidant responses in Arabidopsis thaliana callus. Planta 198, 371-377. doi: $10.1007 /$ BF00620053

Pérez-Pérez, M. E., and Crespo, J. L. (2010). Autophagy in the model alga Chlamydomonas reinhardtii. Autophagy 6, 562-563. doi: 10.4161/auto.6.4.11822

Pérez-Pérez, M. E., Florencio, F. J., and Crespo, J. L. (2010). Inhibition of target of rapamycin signaling and stress activate autophagy in Chlamydomonas reinhardtii. Plant Physiol. 152, 1874-1888. doi: 10.1104/pp.109.152520

Pérez-Pérez, M. E., Couso, I., Heredia-Martínez, L. G., and Crespo, J. L. (2017). Monitoring autophagy in the model green microalga Chlamydomonas reinhardtii. Cells 6, 36. doi: 10.3390/cells6040036

Phadtare, S., and Severinov, K. (2010). RNA remodeling and gene regulation by cold shock proteins. RNA Biol. 7, 788-795. doi: 10.4161/rna.7.6.13482

Phadtare, S. (2004). Recent developments in bacterial cold-shock response. Curr. Issues Mol. Biol. 6, 125-136.

Puyaubert, J., and Baudouin, E. (2014). New clues for a cold case: nitric oxide response to low temperature. Plant Cell Environ. 37, 2623-2630. doi: 10.1111/pce.12329

Renaut, J., Hausman, J.-F., and Wisniewski, M. E. (2006). Proteomics and lowtemperature studies: bridging the gap between gene expression and metabolism. Physiol. Plant 126, 97-109. doi: 10.1111/j.1399-3054.2006.00617.x

Rütgers, M., and Schroda, M. (2013). A role of VIPP1 as a dynamic structure within thylakoid centers as sites of photosystem biogenesis? Plant Signal. Behav. 8, e27037. doi: 10.4161/psb.27037

Rütgers, M., Muranaka, L. S., Schulz-Raffelt, M., Thoms, S., Schurig, J., Willmund, F., et al. (2017). Not changes in membrane fluidity but proteotoxic stress triggers heat shock protein expression in. Chlamydomonas Reinhardtii. Plant Cell Environ. 40, 2987-3001. doi: 10.1111/pce.13060

Saita, E. A., and de Mendoza, D. (2015). Thermosensing via transmembrane protein-lipid interactions. Biochim. Biophys. Acta Biomembr. 1848, 17571764. doi: 10.1016/j.bbamem.2015.04.005

Sanghera, G. S., Wani, S. H., Hussain, W., and Singh, N. B. (2011). Engineering cold stress tolerance in crop plants. Curr. Genomics 12, 30-43. doi: 10.2174/ 138920211794520178

Sawyer, A. L., Landsberg, M. J., Ross, I. L., Kruse, O., Mobli, M., and Hankamer, B. (2015). Solution structure of the RNA-binding cold-shock domain of the Chlamydomonas reinhardtii NAB1 protein and insights into RNA recognition. Biochem. J. 469, 97-106. doi: 10.1042/BJ20150217

Schroda, M., Hemme, D., and Mühlhaus, T. (2015). The Chlamydomonas heat stress response. Plant J. 82, 466-480. doi: 10.1111/tpj.12816

Schulz-Raffelt, M., Lodha, M., and Schroda, M. (2007). Heat shock factor 1 is a key regulator of the stress response in Chlamydomonas. Plant J. 52, 286-295. doi: 10.1111/j.1365-313X.2007.03228.x

Shi, Y., and Yang, S. (2014). "ABA regulation of the cold stress response in plants," in Abscisic Acid: Metabolism, Transport and Signaling. Ed. D.-P. Zhang (Dordrecht: Springer), 337-363. doi: 10.1007/978-94-017-9424-4_17

Siddiqui, K. S., Williams, T. J., Wilkins, D., Yau, S., Allen, M. A., Brown, M. V., et al. (2013). Psychrophiles. Annu. Rev. Earth Planet Sci. 41, 87-115. doi: 10.1146/annurev-earth-040610-133514

Sinetova, M. A., and Los, D. A. (2016). New insights in cyanobacterial cold stress responses: Genes, sensors, and molecular triggers. Biochim. Biophys. Acta Gen. Subj. 1860, 2391-2403. doi: 10.1016/j.bbagen.2016.07.006

Skirycz, A., Bodt, S. D., Obata, T., Clercq, I. D., Claeys, H., Rycke, R. D., et al. (2010). Developmental stage specificity and the role of mitochondrial metabolism in the response of Arabidopsis leaves to prolonged mild osmotic stress. Plant Physiol. 152, 226-244. doi: 10.1104/pp.109.148965

Suzuki, L., and Johnson, C. (2002). Photoperiodic control of germination in the unicell Chlamydomonas. Naturwissenschaften 89, 214-220. doi: 10.1007/ s00114-002-0302-6

Suzuki, N., and Mittler, R. (2006). Reactive oxygen species and temperature stresses: a delicate balance between signaling and destruction. Physiol. Plant 126, 45-51. doi: 10.1111/j.0031-9317.2005.00582.x
Thakur, P., and Nayyar, H. (2013). Facing the cold stress by plants in the changing environment: sensing, signaling, and defending mechanisms in Plant Acclimation to Environ. Stress Eds. N. Tuteja and S. Singh Gill (New York, NY: Springer), 29-69. doi: 10.1007/978-1-4614-5001-6_2

Thalmann, M., and Santelia, D. (2017). Starch as a determinant of plant fitness under abiotic stress. New Phytol. 214, 943-951. doi: 10.1111/nph.14491

Timperio, A. M., Egidi, M. G., and Zolla, L. (2008). Proteomics applied on plant abiotic stresses: role of heat shock proteins (HSP). J. Proteomics 71, 391-411. doi: 10.1016/j.jprot.2008.07.005

Tribelli, P. M., and López, N.II (2018). Reporting key features in cold-adapted bacteria. Life 8, 8. doi: 10.3390/life 8010008

Unal, D., and Cekic, F. O. (2019). Cold acclimation of SnRK2.2 kinases mutant Chlamydomonas reinhardtii. Phycol. Res. 67, 202-207. doi: 10.1111/pre.12371

Valledor, L., Furuhashi, T., Hanak, A.-M., and Weckwerth, W. (2013). Systemic cold stress adaptation of Chlamydomonas reinhardtii. Mol. Cell Proteomics 12, 2032-2047. doi: 10.1074/mcp.M112.026765

Vanlerberghe, G. C. (2013). Alternative oxidase: a mitochondrial respiratory pathway to maintain metabolic and signaling homeostasis during abiotic and biotic stress in plants. Int. J. Mol. Sci. 14, 6805-6847. doi: 10.3390/ijms14046805

Vashisht, A. A., and Tuteja, N. (2006). Stress responsive DEAD-box helicases: a new pathway to engineer plant stress tolerance. J. Photochem. Photobiol. 84, 150-160. doi: 10.1016/j.jphotobiol.2006.02.010

Vaultier, M.-N., Cantrel, C., Vergnolle, C., Justin, A.-M., Demandre, C., Benhassaine-Kesri, G., et al. (2006). Desaturase mutants reveal that membrane rigidification acts as a cold perception mechanism upstream of the diacylglycerol kinase pathway in Arabidopsis cells. FEBS Lett. 580, 42184223. doi: 10.1016/j.febslet.2006.06.083

Wang, X., Chang, L., Wang, B., Wang, D., Li, P., Wang, L., et al. (2013). Comparative proteomics of Thellungiella halophila leaves from plants subjected to salinity reveals the importance of chloroplastic starch and soluble sugars in halophyte salt tolerance. Mol. Cell Proteomics 12, $2174-$ 2195. doi: $10.1074 /$ mcp.M112.022475

Wang, Y., Zhou, J., and Yu, J. (2017). The critical role of autophagy in plant responses to abiotic stresses. Front. Agr. Sci. Eng. 4, 28-36. doi: 10.15302/j-fase2017130

Wei, L., Derrien, B., Gautier, A., Houille-Vernes, L., Boulouis, A., Saint-Marcoux, D., et al. (2014). Nitric oxide-triggered remodeling of chloroplast bioenergetics and thylakoid proteins upon nitrogen starvation in Chlamydomonas reinhardtii. Plant Cell. 26, 353-372. doi: 10.1105/tpc.113.120121

Xie, Z., Nolan, T. M., Jiang, H., and Yin, Y. (2019). AP2/ERF transcription factor regulatory networks in hormone and abiotic stress responses in Arabidopsis. Front. Plant Sci. 10, 228. doi: 10.3389/fpls.2019.00228

Zalutskaya, Z., Skryabina, U. S., and Ermilova, E. V. (2019). Generation of hydrogen peroxide and transcriptional regulation of antioxidant enzyme expression in Chlamydomonas reinhardtii under hypothermia. Russ. J. Plant Physiol. 66, 223-230. doi: 10.1134/S1021443719020171

Zheng, Y., Xue, C., Chen, H., He, C., and Wang, Q. (2020). Low-temperature adaptation of the snow alga Chlamydomonas nivalis is associated with the photosynthetic system regulatory process. Front. Microbiol. 11, 1233. doi: $10.3389 /$ fmicb.2020.01233

Zhu, X., Liu, S., Sun, L., Song, F., Liu, F., and Li, X. (2018). Cold tolerance of photosynthetic electron transport system is enhanced in wheat plants grown under elevated $\mathrm{CO}_{2}$. Front. Plant Sci. 9, 933-940. doi: 10.3389/fpls.2018.00933

Conflict of Interest: The author declares that the research was conducted in the absence of any commercial or financial relationships that could be construed as a potential conflict of interest.

Copyright $\odot 2020$ Ermilova. This is an open-access article distributed under the terms of the Creative Commons Attribution License (CC BY). The use, distribution or reproduction in other forums is permitted, provided the original author(s) and the copyright owner(s) are credited and that the original publication in this journal is cited, in accordance with accepted academic practice. No use, distribution or reproduction is permitted which does not comply with these terms. 